\title{
A Life Saving Emergency Department Resuscitative Endovascular Balloon Occlusion of the Aorta (REBOA) with Open Groin Technique
}

\author{
Panu Teeratakulpisarn (D) \\ Phati Angkasith \\ Parichat Tanmit (D) \\ Chaiyut Thanapaisal \\ Supatcha Prasertcharoensuk \\ Narongchai Wongkonkitsin \\ Department of Surgery, Faculty of \\ Medicine, Khonkaen University, Khon \\ Kaen, Thailand
}

\begin{abstract}
A 53-year-old male pedestrian was hit by a car and arrived at our hospital with a blunt abdominal injury and hemorrhagic shock. Resuscitative Endovascular Balloon Occlusion of the Aorta (REBOA) was performed in a timely fashion using the open groin technique in the emergency room. The procedure resulted in rapid improvement of hemodynamic status while the bleeding source was controlled. Recently, REBOA is a proper adjunctive procedure in major non-compressible torso hemorrhage patients. The procedure requires a portable X-ray or fluoroscopic machine in the ER to confirm the balloon's position. This method has likely limited the use of REBOA in developing countries. The procedure with open groin technique, using anatomical landmarks and physiologic change to confirm the position of the balloon, has been developed to address these concerns. Here we report on the treatment's success with this technique and believe that it can benefit trauma patient care.
\end{abstract}

Keywords: REBOA, torso hemorrhage, shock, abdominal trauma, resuscitation, balloon

\section{Introduction}

Non-compressible torso hemorrhage is a significant cause of death in trauma patients, which can be prevented. ${ }^{1}$ These types of injuries include pelvic fractures, laceration, or rupture of intraabdominal or retroperitoneal organs. Bleeding control in these areas is time-consuming. ${ }^{2-5}$

The temporary bleeding control in a hemorrhagic shock patient from noncompressible torso hemorrhage can be performed by resuscitative thoracotomy with aortic cross-clamping. After this procedure, There is time for resuscitation and transfer the patient to the operating room. ${ }^{6}$ Disadvantage of this procedure is low survival rate and complications. ${ }^{7-9}$

At present, endovascular procedures have significantly evolved. The Resuscitative Endovascular Balloon Occlusion of the Aorta (REBOA) is widely used to manage hemorrhagic shock in trauma patients. This procedure can be performed quickly. Currently, patients' survival rate with this procedure is more significant than those treated with resuscitative thoracotomy. ${ }^{10}$

The clinical use of REBOA is similar to resuscitative thoracotomy, which is traumatic, life-threatening hemorrhage below the diaphragm in patients in hemorrhagic shock who are refractory to resuscitation, the cardiac arrest patient due to non-compressible torso hemorrhage below the diaphragm within 10 minutes with
Correspondence: Panu Teeratakulpisarn Department of Surgery, Faculty of Medicine, Khonkaen University, I23,

Moo 16, Mittraphap Road, Nai-Muang

Muang District, Khon Kaen, 40002,

Thailand

Tel +6686637/ 234

Email panute@kku.ac.th 
adequate cardiopulmonary resuscitation or the noncompressible torso hemorrhage who does not respond to volume resuscitation. ${ }^{11}$ However, after performing this procedure, the patient should not be delayed to receive the definite hemorrhage control. ${ }^{12}$

The balloon's positioning in the aorta, the wire should be advanced under fluoroscopic guidance or visualization such that the floppy tip is in the distal aortic arch. ${ }^{13}$ This action, which makes it very time-consuming. The majority of our hospitals in Thailand have limited resources for this ER procedure, such as the fluoroscopic machine or the proper REBOA commercial kit. Also, there are no vascular surgeons in every provincial hospital to perform the complex procedure. Here, we present a case of blunt abdominal injury with hemorrhagic shock treated using Resuscitative Endovascular Balloon Occlusion of the Aorta (REBOA) with open groin technique and confirmed the position of the balloon using external landmarks and clinical presentation. We also report the clinical outcome of this technique. To our knowledge, this technique is rarely mentioned.

\section{Case Report}

A 53-year-old male pedestrian was hit by a car 20 minutes before arriving at the hospital. Upon the arrival of the emergency medical services, the patient was drowsy but following commands. While being taken to the hospital, the patient became hypotensive. He was brought by ambulance to our trauma center under ongoing resuscitative measures. Anywise, no intravenous access was established yet. After the primary survey following the ATLS ${ }^{\circledR}$ protocol, there were distended abdomen and left fibular fractured. The extended Focused Assessment Sonography in Trauma (eFAST) revealed free fluid in the peritoneal cavity. His pulse rate was 142 beats per minute, and his blood pressure was $66 / 43$ millimeters of mercury by the upper arm cuff at ER.

The patient was intubated, and a massive transfusion protocol was activated immediately. The right common femoral artery was cut down, and insert a 12-French gauge introducer vascular sheath (Figure 1). The conventional (over the wire) aortic balloon was inserted via introducer vascular sheath over the guidewire (Figure 2). The length of the balloon was measured by the distance between the right groin and mid sternum (Figure 3). After inflation of the aortic balloon, blood pressure was raised to $118 / 78$ millimeters of mercury. Confirmation of the aortic balloon was made by the physiologic response of the patient with there is

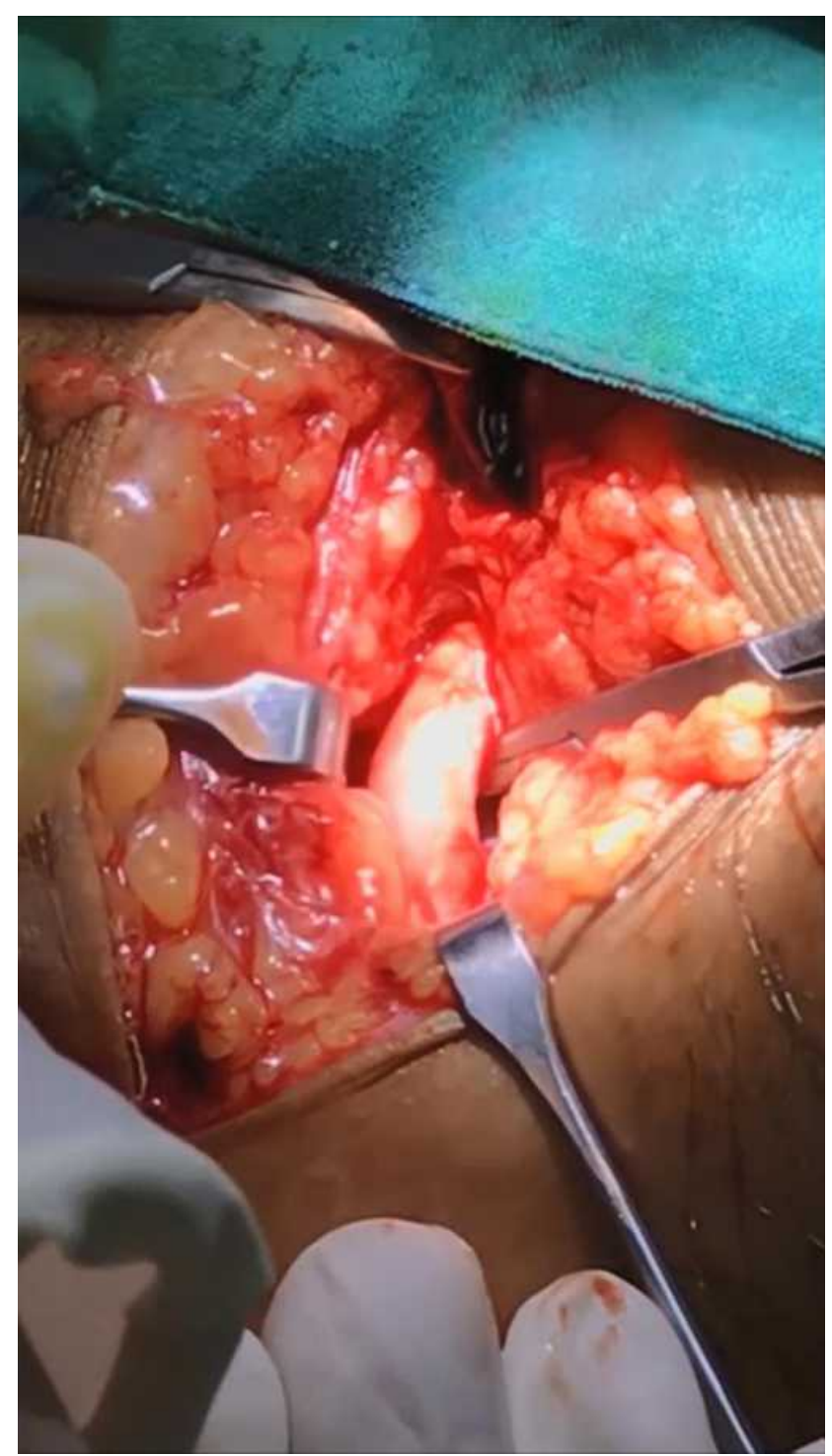

Figure I Right groin vertical incision to identify right common femoral artery and prepare for insert the vascular sheath.

no pulse palpated in both lower extremities and the blood pressure in upper extremities is raised. We used complete aortic occlusion technique in this patient.

The patient was sent to the operating room for immediate laparotomy. There was a sizeable mesenteric avulsion and 10 centimeters of jejunal contusion, causing 2500 millimeters of hemoperitoneum (Figure 4). Segmental small bowel resection with end-to-end anastomosis of jejunum and suture to stop bleeding from mesenteric vessels were performed. At the same time, the patient was resurrected with medication and blood components by the anesthesiologists. The intraabdominal procedures took 55 minutes, and then we tried to deflate the balloon. However, when we deflated the balloon, the blood pressure dropped immediately. So, we inflated the 


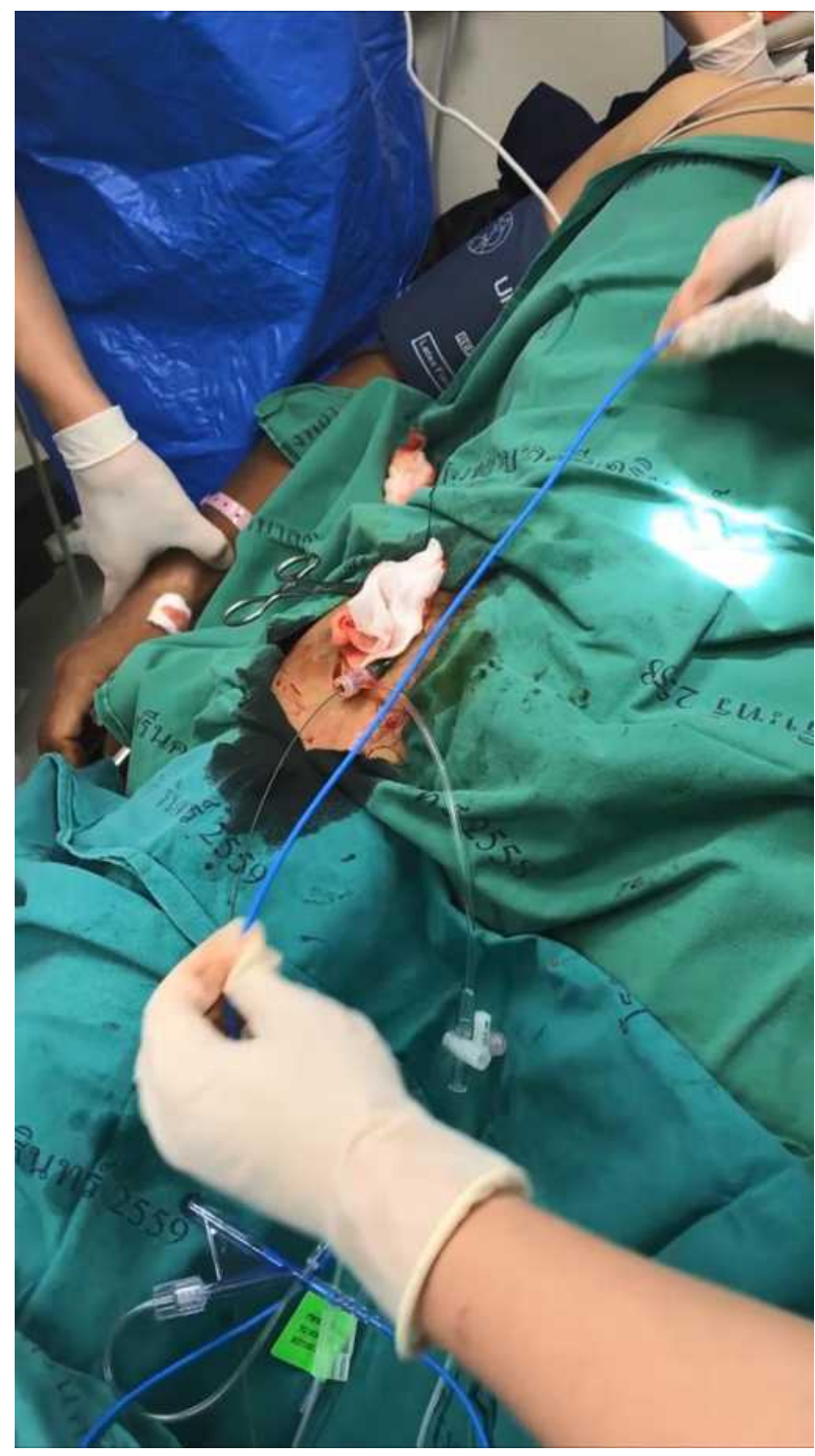

Figure 2 The Aortic Balloon was being inserted into the right Common Femoral artery via the vascular sheath.

balloon for the anesthesiologist to resuscitated the patient. After that, we deflated the balloon again. The total aortic occlusion time took 90 minutes. The patient's blood pressure was approximately 120/75 millimeters of mercury by arterial-line after the operation.

After the hemodynamic was stable, the right common femoral artery was repaired, and the wound was sutured. Both dorsalis pedis pulses were palpated.

The total operative time was 140 minutes. The patient received ten units PRBCs, eight units of fresh-frozen plasma, and six units of platelets.

All of the procedures were performed by a trauma surgeon trained in vascular procedures on duty that day and covered by the vascular surgical staff.

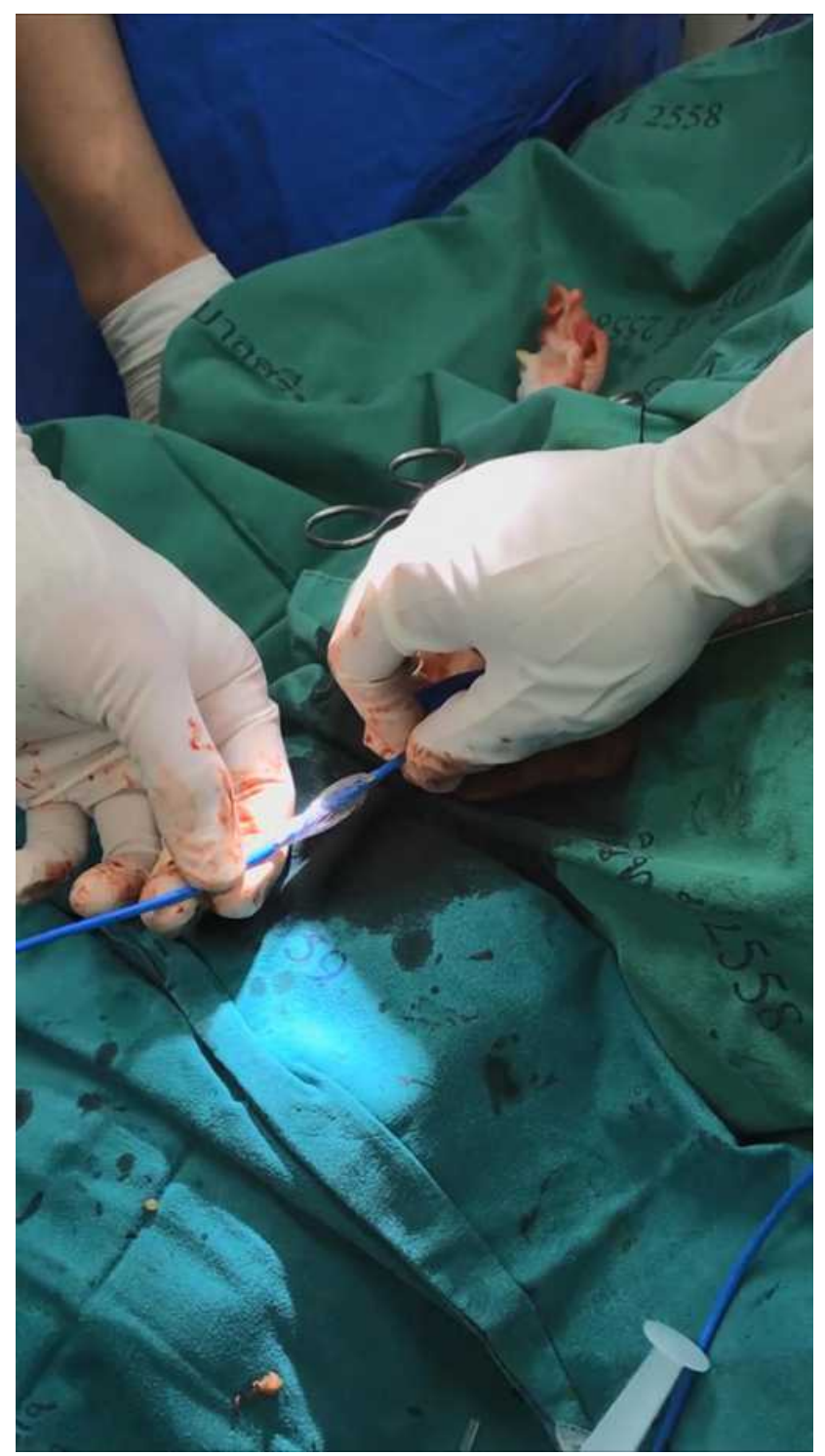

Figure 3 The length of the aortic balloon was being measured by the distance between the right groin and mid sternum to ensure the balloon was placed in the Zone I of the Aorta.

The patient did well and was discharged on hospital day 5. He required rehabilitation and ambulation training. The telephone followed up to track symptoms and found that the patient did not have any eating problems.

\section{Discussion}

The REBOA is a proper adjunctive procedure in major hemorrhage patients. However, there are some limitations and should be performed only by experienced physicians or surgeons aware of the access site complications and can immediately perform surgical treatment. ${ }^{11}$ The patients undergoing REBOA have improved overall survival than the patients undergoing resuscitative thoracotomy. ${ }^{14}$ This 


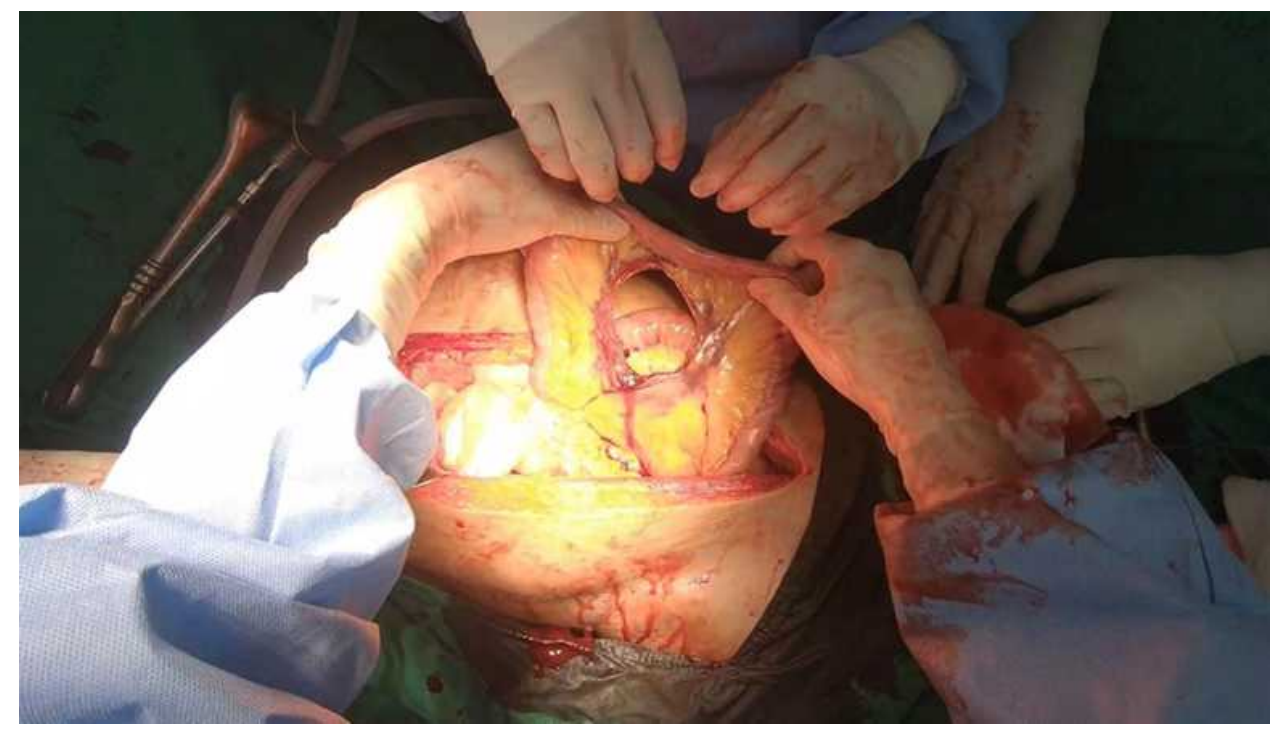

Figure 4 The mesenteric avulsion of the jejunum with active bleeding from the mesenteric vessels.

procedure helps abdominal and pelvic trauma, ruptured aortic aneurysm, gastrointestinal hemorrhage, and postpartum hemorrhage. ${ }^{15}$ There is a meta-analysis found that there was $4-5 \%$ of groin access associated complication after the use of REBOA. ${ }^{16}$ Another meta-analysis also mentions the use of REBOA in management for significant hemorrhage, 89 articles and 28 case reports found that REBOA increases systolic blood pressure in hemorrhagic shock patient as an adjunct for definitive surgery in hemodynamic instability with an iatrogenic vessel injury during approach below $5 \%{ }^{2}$

In our case, it was necessary to perform a surgical cut down of the common femoral artery, which can be easier to puncture and insert the femoral sheath if the patient has weak or no pulse. The procedure can be done quickly by surgeons familiar with the groin anatomy. There was a study using the mid-sternum landmark for placement of the balloon in the landing zone was 100\% accuracy with an acceptable margin of safety. ${ }^{17}$ To confirm the effectiveness of the balloon placement, the mean arterial pressure of both upper extremities should increase and the pulses below the location of the balloon should be impalpable.

Studies have shown that the use of smaller vascular sheaths appeared to have fewer complications such as distal limb ischemia or the need to re-anastomosis of the vessels. Despite relatively lengthy procedures and requiring external compression on removal were noted. ${ }^{18,19}$

Another difference in the open groin technique is that it is necessary to repair the common femoral artery and the groin incision. Nevertheless, this step should be done after the major operation, and the patient's hemodynamic is stable.

The major problem of developing countries is inadequate equipment in the emergency department, and also the portable radiography quite take time. This open groin technique should be another way to save the patient's life in the limited resources situation. This open groin technique has an advantage over the conventional REBOA technique in terms of cost-effectiveness and limited resource use while providing the same therapeutic results.

Another problem is that Thailand is unable to claim reimbursement for commercial REBOA operations. So we try to find ways to help our patients get the most out of them by being used as much equipment as available to treat the patient (such as the reusable aortic balloon and vascular sheath).

\section{Conclusion}

Emergency department REBOA is an effective adjunct procedure for controlling major abdominal and pelvic hemorrhage patients, which can provide at least similar results to the resuscitative thoracotomy. The open groin technique with using of physiologic change of the patient to confirm the effectiveness of the emergency department REBOA can be safe to perform, which should be helpful in developing countries or small hospitals without the fluoroscopic machine in the resuscitation room. 
Further study with a more comparable population, patient factors, and hospital resources is needed to define the application of open groin technique REBOA.

\section{Ethics Approval and Consent to Participate}

Center for Ethics in Human Research of Khon Kaen University (KKUEC) approved this case report and the committee's reference number is HE631389.

\section{Consent for Publication}

The patient informed consent for publish the case details and images.

\section{Acknowledgments}

All authors would like to thank (a) Associate Professor Polpun Boonmak for resuscitation and performed anesthesia of the patient and (b) all emergency nurses in the Emergency Department, Khon Kaen University, Thailand helping the procedures.

\section{Disclosure}

The authors declare that there are no conflicts of interest regarding the publication of this article.

\section{References}

1. Mill V, Wellme E, Montán C. Trauma patients eligible for resuscitative endovascular balloon occlusion of the aorta (REBOA), a retrospective cohort study. Eur J Trauma Emerg Surg. 2020. doi:10.1007/s00068020-01345-w

2. Borger van der Burg BLS, van Dongen TTCF, Morrison JJ, et al. A systematic review and meta-analysis of the use of resuscitative endovascular balloon occlusion of the aorta in the management of major exsanguination. Eur $J$ Trauma Emerg Surg. 2018;44 (4):535-550. doi:10.1007/s00068-018-0959-y

3. Gamberini E, Coccolini F, Tamagnini B, et al. Resuscitative endovascular balloon occlusion of the aorta in trauma: a systematic review of the literature. World J Emerg Surg. 2017;12(1):42. doi:10.1186/ s13017-017-0153-2

4. Saito N, Matsumoto H, Yagi T, et al. Evaluation of the safety and feasibility of resuscitative endovascular balloon occlusion of the aorta. J Trauma Acute Care Surg. 2015;78(5):897-904. doi:10.1097/ TA.0000000000000614

5. Ribeiro Júnior MAF, Maurício AD, Costa CTK, et al. Expanding indications and results for the use of resuscitative endovascular balloon occlusion of the aorta - REBOA. Rev Col Bras Cir. 2019;46(5): e20192334. doi:10.1590/0100-6991e-20192334

6. Manzano Nunez R, Naranjo MP, Foianini E, et al. A meta-analysis of resuscitative endovascular balloon occlusion of the aorta (REBOA) or open aortic cross-clamping by resuscitative thoracotomy in non-compressible torso hemorrhage patients. World J Emerg Surg. 2017;12(1):30. doi:10.1186/s13017-017-0142-5
7. Suliburk JW. Complications of emergency center thoracotomy. Tex Heart Inst J. 2012;39(6):876-877.

8. Søreide K, Petrone P, Asensio JA. Emergency thoracotomy in trauma: rationale, risks, and realities. Scand J Surg. 2007;96 (1):4-10. doi:10.1177/145749690709600102

9. Nevins EJ, Bird NTE, Malik HZ, et al. A systematic review of 3251 emergency department thoracotomies: is it time for a national database? Eur $J$ Trauma Emerg Surg. 2019;45(2):231-243. doi:10.1007/s00068-018-0982-z

10. Otsuka H, Sato T, Sakurai K, et al. Effect of resuscitative endovascular balloon occlusion of the aorta in hemodynamically unstable patients with multiple severe torso trauma: a retrospective study. World J Emerg Surg. 2018;13(1):49. doi:10.1186/s13017-018-0210-5

11. Bulger EM, Perina DG, Qasim Z, et al. Clinical use of resuscitative endovascular balloon occlusion of the aorta (REBOA) in civilian trauma systems in the USA, 2019: a joint statement from the American College of Surgeons Committee on Trauma, the American College of Emergency Physicians, the National Association of Emergency Medical Services Physicians and the National Association of Emergency Medical Technicians. Trauma Surg Acute Care Open. 2019;4(1):e000376. doi:10.1136/tsaco-2019000376

12. Matsumura Y, Matsumoto J, Kondo H, et al. Early arterial access for resuscitative endovascular balloon occlusion of the aorta is related to survival outcome in trauma. J Trauma Acute Care Surg. 2018;85 (3):507-511. doi:10.1097/TA.0000000000002004

13. Stannard A, Eliason JL, Rasmussen TE. Resuscitative Endovascular Balloon Occlusion of the Aorta (REBOA) as an adjunct for hemorrhagic shock. $J$ Trauma. 2011;71(6):1869-1872. doi:10.1097/ TA.0b013e31823fe 90c

14. Moore LJ, Brenner M, Kozar RA, et al. Implementation of resuscitative endovascular balloon occlusion of the aorta as an alternative to resuscitative thoracotomy for noncompressible truncal hemorrhage. J Trauma Acute Care Surg. 2015;79(4):523-532. doi:10.1097/ TA.0000000000000809

15. Morrison JJ, Galgon RE, Jansen JO, Cannon JW, Rasmussen TE, Eliason JL. A systematic review of the use of resuscitative endovascular balloon occlusion of the aorta in the management of hemorrhagic shock. J Trauma Acute Care Surg. 2016;80(2):324-334. doi:10.1097/TA.000000000000913

16. Manzano-Nunez R, Orlas CP, Herrera-Escobar JP, et al. A meta-analysis of the incidence of complications associated with groin access after the use of resuscitative endovascular balloon occlusion of the aorta in trauma patients. $J$ Trauma Acute Care Surg. 2018;85(3):626-634. doi:10.1097/TA.0000000000001978

17. Linnebur M, Inaba $\mathrm{K}$, Haltmeier $\mathrm{T}$, et al. Emergent non-imageguided resuscitative endovascular balloon occlusion of the aorta (REBOA) catheter placement: a cadaver-based study. J Trauma Acute Care Surg. 2016;81(3):453-457. doi:10.1097/ TA.0000000000001106

18. Matsumura Y, Matsumoto J, Kondo H, et al. Fewer REBOA complications with smaller devices and partial occlusion: evidence from a multicentre registry in Japan. Emerg Med J. 2017;34 (12):793-799. doi:10.1136/emermed-2016-206383

19. Ordoñez CA, Khan M, Cotton B, et al. The Colombian experience in Resuscitative Endovascular Balloon Occlusion of the Aorta (REBOA): the progression from a large caliber to a low-profile device at a Level I Trauma Center. Shock. 2020. doi:10.1097/ SHK.0000000000001515 


\section{Publish your work in this journal}

The Open Access Emergency Medicine is an international, peerreviewed, open access journal publishing original research, reports, editorials, reviews and commentaries on all aspects of emergency medicine. The manuscript management system is completely online and includes a very quick and fair peer-review system, which is all easy to use. Visit http://www.dovepress.com/testimonials.php to read real quotes from published authors.

Submit your manuscript here: https://www.dovepress.com/open-access-emergency-medicine-journal 